\title{
Nanocompósitos de poli(álcool vinílico) contendo materiais híbridos mimetizando o pigmento Azul Maya
}

\section{Poly(vinyl alcohol) nanocomposites containing hybrid materials mimicking the Maya Blue pigment}

\author{
Vicente Lira Kupfer ${ }^{1}$, Silvia Jaerger ${ }^{1}$ e Fernando Wypych ${ }^{1 *}$ \\ 'Laboratório de Química de Materiais Avancados - LAQMA, Departamento de Química, \\ Centro de Pesquisa em Química Aplicada - CEPESQ, Universidade Federal do Paraná - UFPR, \\ Curitiba, PR, Brazil \\ *wypych@ufpr.br
}

\begin{abstract}
Resumo
De forma a mimetizar o pigmento Azul Maya, dois corantes azo (alaranjado de metila - AM e vermelho congo - VC) foram inseridos na estrutura porosa da paligorsquita. Os materiais híbridos foram caracterizados por difração de raios X, espectroscopia de energia dispersiva, microscopia eletrônica de varredura, espectroscopia na região do ultravioleta-visível e análise superficial (área superficial por BET, tamanho e volume de poros por BJH). Após dispersar os materiais híbridos no poli(álcool vinílico), filmes coloridos e transparentes foram obtidos por casting úmido. Após acondicionamento em uma dessecador por uma semana a uma umidade controlada de $43 \pm 2 \%$ os filmes foram avaliados em relação as suas propriedades mecânicas. De modo geral, o material PVA-PGS/AM teve um aumento no módulo de Young, tensão de ruptura e redução do alongamento enquanto que a paligorsquita bruta e PVA-PGS/VC apresentaram um comportamento oposto.
\end{abstract}

Palavras-chave: corante azo, materiais híbridos, propriedades mecânicas, paligorsquita, poli(álcool vinílico).

\begin{abstract}
To mimic the Maya Blue pigment, two azo dyes (methylorange - AM and congo red - VC) were inserted into the porous structure of paligorskite. The hybrid materials were characterized by X-ray diffraction, energy dispersive spectroscopy, scanning electron microscopy, UV-Vis spectroscopy and surface analysis (surface area by BET, pore size and volume by and BJH). After dispersing the hybrid materials into poly(vinyl alcohol), colored and transparent films were obtained by wet casting. After conditioned into desiccators at controlled humidity of $43 \pm 2 \%$ for one week, the films were evaluated in relation to their mechanical properties. In general, the material PVA-PGS/AM increases Young's modulus and ultimate tensile strength reducing the elongation while raw paligorskite and PVA-PGS/VC presented an opposite behavior.
\end{abstract}

Keywords: azo dyes, hybrid materials, mechanical properties, palygorskite, poly(vinyl alcohol).

\section{Introdução}

O surgimento de novas tecnologias exige uma demanda por materiais com combinações incomuns de suas propriedades, as quais não podem ser alcançadas somente com suas características individuais. A combinação de compostos orgânicos ou inorgânicos com materiais poliméricos origina os compósitos poliméricos, os quais tem contribuído de forma importante no desenvolvimento de novos materiais para aplicações variadas, especialmente na indústria esportiva, automobilística e aeroespacial. Os primeiros nanocompósitos poliméricos foram desenvolvidos na década de 90 pelo grupo de pesquisas da Toyota Motors ${ }^{\circledR}$ do Japão, nos quais foram utilizados como cargas, argilominerais trocadores catiônicos de grupo 2:1 hidrofobizados. Argilominerais lamelares "in natura" ou modificados por reações de troca iônica, quando inseridos em matrizes poliméricas, geralmente dão origem a materiais com melhores propriedades ópticas, térmicas, elétricas, mecânicas, etc. ${ }^{[1-6]}$

Na maioria das aplicações citadas acima, a superfície dos sólidos desempenha um papel fundamental, de modo que a reatividade está diretamente relacionada à composição química, morfologia e características superficiais. A maioria dos trabalhos da literatura utiliza materiais lamelares naturais e/ou sintéticos especialmente no intuito de produzir materiais híbridos para serem utilizados como materiais funcionais, porém materiais fibrosos nanoestruturados também têm atraído a atenção dos pesquisadores.

Nesse contexto utilizou-se a paligorsquita que é um filossilicato de magnésio e alumínio com fórmula idealizada $\mathrm{Mg}_{2} \mathrm{Al}_{2} \mathrm{Si}_{8} \mathrm{O}_{20}(\mathrm{OH})_{2}\left(\mathrm{H}_{2} \mathrm{O}\right)_{4} \cdot 4 \mathrm{H}_{2} \mathrm{O}$. Seu uso está ligado à obtenção do pigmento Azul Maya, um híbrido formado 
entre a paligorsquita e o corante índigo, utilizado em tempos pré-coloniais na Península de Yucatán no México ${ }^{[7]}$. Uma das principais características desse material é promover uma maior estabilidade das moléculas do corante índigo que são introduzidas nos canais nanométricos da paligorsquita, as quais resistem à ataques com soluções ácidas e/ou alcalinas diluídas, tratamento com solventes e exposição intensa à luz ${ }^{[8,9]}$. Essas características fizeram com que pinturas utilizando o pigmento Azul Maya resistissem às imtempéries por mais de 1200 anos.

Outros argilominerais lamelares como a caulinita, montmorilonita e nontronita contendo o corante índigo também foram investigados com o objetivo de produzir pigmentos estáveis, no entanto não apresentaram resultados comparáveis à paligorsquita. Além de várias artigos reportando a interação de outros corantes na paligorsquita ${ }^{[10-13]}$, testes também foram realizados com o análogo da paligorsquita, a sepiolita, a qual demonstrou uma menor estabilidade química devido à sua pronunciada fragilidade estrutura ${ }^{[14]}$. Outras matrizes lamelares sintéticas como os hidróxidos duplos lamelares (HDL) e os hidroxissais lamelares (HSL) também foram intercaladas com corantes aniônicos azo e os materiais dispersos em polímeros, porém estudos de estabilidade química em longo prazo não foram reportados ${ }^{[15-18]}$.

Devido à constatação de novas possibilidades de obtenção de pigmentos híbridos entre paligorsquita e corantes azo e a inserção desse material híbrido em matrizes poliméricas formando um material nanocompósito multifuncional, abre-se uma nova perspectiva de estudos das propriedades desses materiais. Entre a vasta gama de polímeros hidrofílicos utilizados nas diversas pesquisas, o poli(álcool vinílico) (PVA) tem atraído a atenção da comunidade científica principalmente pela fácil obtenção de películas altamente transparentes e com excelentes propriedades mecânicas e térmicas, além de uma boa interação com materiais hidrofóbicos ou hidrofílicos e até mesmo metais.

Baseado nesses antecedentes, os estudos realizados neste trabalho consistem na obtenção de materiais híbridos orgânicos/inorgânicos, utilizando a paligorsquita como matriz inorgânica para a imobilização de corantes azo. Os materiais híbridos foram utilizados como cargas em uma matriz polimérica de PVA, obtendo os materiais nanocompósitos poliméricos empregando a técnica de "casting" úmido. Os materiais híbridos e os filmes dos nanocompósitos foram caracterizados por diferentes técnicas instrumentais objetivando avaliar suas propriedades químicas, térmicas, mecânicas e estruturais.

\section{Materiais e Métodos}

Os sais de sódio dos corantes azo utilizados foram o alaranjado de metila $\left(\mathrm{C}_{14} \mathrm{H}_{14} \mathrm{~N}_{3} \mathrm{NaO}_{3} \mathrm{~S}\right)$ (Figure 1a) e o vermelho congo $\left(\mathrm{C}_{32} \mathrm{H}_{22} \mathrm{~N}_{6} \mathrm{Na}_{2} \mathrm{O}_{6} \mathrm{~S}_{2}\right)$ (Figure $1 \mathrm{~b}$ ), ambos da marca Vetec. A escolha dos corantes do grupo azo se deu através da dimensão das moléculas e dimensões dos poros da paligorsquita, sendo que o ânion derivado do AM poderia ser totalmente inserido nos poros enquanto que $\mathrm{o}$ ânion do VC poderia ser parcialmente inserido. Além das dimensões, foram escolhidos sais de sódio que conferem a

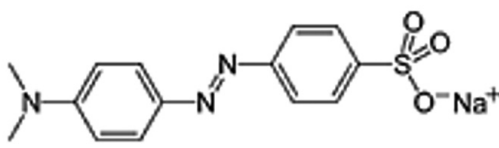

(a)

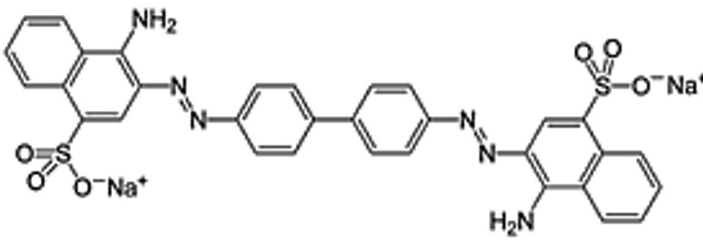

(b)

Figura 1. Estrutura dos corantes azo na forma de sais de sódio utilizado na preparação dos materiais híbridos. Alaranjado de metila - AM (a) e vermelho congo - VC (b).

solubilidade em água, possibilitando diferentes interações de absorção ou adsorção com a paligorsquita.

\subsection{Sintese dos materiais híbridos}

Nas sínteses dos materiais híbridos utilizou-se paligorsquita PFI-1 $\left(\mathrm{Mg}_{0,33} \mathrm{Ca}_{0.62} \mathrm{Na}_{0.04} \mathrm{~K}_{0,13}\right)\left[\mathrm{Al}_{1.50} \mathrm{Fe}^{(\mathrm{III})}{ }_{0.52} \mathrm{Fe}^{\text {(II) }}{ }_{0.01} \mathrm{Mn}_{0.01} \mathrm{Mg}_{1.91}\right.$ $\left.\mathrm{Ti}_{0.06}\right]\left[\mathrm{Si}_{7.88} \mathrm{Al}_{0.22}\right] \mathrm{O}_{20}(\mathrm{OH})_{4}$, fornecida pelo repositório da Clay Minerals Society, Universidade de Purdue (Gadsden County, Florida, USA).

Os materiais foram obtidos por síntese mecanoquímica, utilizando-se gral e pistilo de ágata. A paligorsquita "in-natura" teve um tratamento prévio de secagem em uma estufa a vácuo, com aquecimento à $120^{\circ} \mathrm{C}$ e vácuo de $-400 \mathrm{~mm}$ de $\mathrm{Hg}$ por 24 horas. Este tratamento foi realizado com intuito de ativar os poros nanométricos da paligorsquita onde estão localizadas as moléculas de água estruturais.

Após estudos de otimização da concentração de corante na matriz mineral, o procedimento de síntese do material híbrido consistiu em misturar a paligorsquita seca $\left(120^{\circ} \mathrm{C}\right.$ por um período de 24 horas) com $4 \%$ em massa dos corantes em relação à massa da paligorsquita e posterior adição de $2 \mathrm{~mL}$ de uma solução $0,5 \mathrm{~mol} / \mathrm{L}$ de $\mathrm{HCl}$. A mistura foi moída por 15 min e submetida a um tratamento térmico em estufa com uma rampa de aquecimento inicial de $20^{\circ} \mathrm{C} /$ hora até $125^{\circ} \mathrm{C}$, adicionando-se $2 \mathrm{~mL}$ da solução ácida $(\mathrm{HCl} 0,5 \mathrm{~mol} / \mathrm{L})$ a cada intervalo de uma hora. $\mathrm{O}$ tratamento final consistiu em tratar a mistura a $125^{\circ} \mathrm{C}$ por um período de 12 horas. Em seguida, o sólido foi moído por 15 min e o excesso dos sais de sódio dos corantes azo foi removido por extração em um sistema Soxhlet com $200 \mathrm{~mL}$ de mistura etanol/água 9:1 (v/v) por um período de 24 horas. Os sólidos resultantes foram secos em estufa à vácuo a $60{ }^{\circ} \mathrm{C} \mathrm{e}-400 \mathrm{~mm}$ de $\mathrm{Hg}$ por 24 horas. Após tentativas infrutíferas de imobilizar os sais de sódio, verificou-se que o processo de protonação é indispensável para um bom processo de imobilização dos corantes azo na estrutura da paligorsquita.

Os materiais foram denominados: PGS-AM (Paligorsquita com o corantes alaranjado de metila), PGS-VC (Paligorsquita com o corante vermelho congo) e os materiais de partida PGS (Paligorsquita) e os corantes na forma de sais de sódio: Na-AM (Alaranjado de Metila) e Na-VC (Vermelho Congo). 


\subsection{Síntese dos nanocompósitos}

Os filmes de PVA foram preparados pelo método de "casting" úmido com diferentes proporções $(1,2,3,5,8 \%)$ em massa de material híbrido ou argilomineral em relação à massa do polímero que foi fixada em $700 \mathrm{mg}$. O polímero utilizado para a preparação dos nanocompósitos foi o poli(álcool vinílico) - PVA (MM = $72000 \mathrm{~g} \mathrm{~mol}^{-1}$, grau de hidrólise de 98\%), com grau de pureza analítico, fornecido pela Merck.

Os materiais híbridos foram suspensos em $5 \mathrm{~mL}$ de etanol e submetidos a tratamento com um banho de ultrassom por uma hora, no intuito de desagregar e dispersar melhor as nanofibras. Após este período, a dispersão da carga foi vertida em um béquer contendo o PVA previamente disperso em $25 \mathrm{~mL}$ de água à $85^{\circ} \mathrm{C}$, e a mistura mantida em agitação magnética vigorosa por $30 \mathrm{~min}$. Em seguida, a mistura foi transferida para uma placa de Petri de aço inox (diâmetro de $6 \mathrm{~cm}$ ) e o solvente foi evaporado à $60^{\circ} \mathrm{C}$ por um período de $24 \mathrm{~h}$ em uma estufa à vácuo operando à $-400 \mathrm{~mm}$ de $\mathrm{Hg}$.

\subsection{Métodos de caracterização}

As medidas de difração de raios $\mathrm{X}$ (DRX) dos materiais híbridos e dos precursores foram conduzidas em porta-amostras de vidro, utilizando-se um difratômetro Shimadzu modelo XRD-6000 com fonte de cobre $\left(\mathrm{CuK}_{\alpha}\right.$, $\lambda=1,5418 \AA$ ) , corrente de $20 \mathrm{~mA}$, tensão de $40 \mathrm{kV}$, velocidade de varredura de $0,5^{\circ} \mathrm{min}^{-1}$ e passo de 0,02 graus. As medidas dos nanocompósitos foram feitas em porta-amostras de alumínio, os quais foram fixados nas laterais por uma fita adesiva. Os espectros eletrônicos na região do UV-Vis foram registrados em espectrofotômetro UV-Vis da Perkin Elmer, modelo Lambda 650, na região entre 200 e $800 \mathrm{~nm}$. Os pHs das soluções dos corantes foram controladas com soluções de $\mathrm{HCl}$ ou $\mathrm{NaOH} 0,1 \mathrm{~mol} / \mathrm{L}$.

Os espectros UV-Vis em modo de refletância difusa foram coletados em um espectrômetro Varian Cary 100 na faixa de 200 a $800 \mathrm{~nm}$.

As isotermas de adsorção de nitrogênio foram obtidas em um analisador de sorção de gás QUANTACHROME, modelo NOVA 2000e. As amostras foram degaseificadas à $120{ }^{\circ} \mathrm{C}$ sob vácuo durante três horas e as análises foram conduzidas sob temperatura de nitrogênio liquido $\left(-196{ }^{\circ} \mathrm{C}\right)$. As áreas superficiais específicas das amostras foram calculadas utilizando o método com pontos múltiplos de Brunauer-Emmet-Teller (BET). As análises de área externa foram obtidas pelo método de t-plot e as análises de volume de poros foram obtidas de acordo com o método Barrett-Joyner-Halenda (BJH).

As imagens dos materiais foram obtidas por microscopia eletrônica de varredura JEOL modelo (JSM-6360LV). As amostras dos filmes foram preparadas por meio de uma fratura criogênica com nitrogênio liquido $\left(-196^{\circ} \mathrm{C}\right)$ e depositada sobre os porta-amostras com fita adesiva de cobre, deixando exposta a região de que ocorreu a fratura.

As análises químicas dos materiais híbridos e dos nanocompósitos foram obtidas pela técnica de espectroscopia de energia dispersiva (EDS), utilizando-se um equipamento Thermo Noran e um Software Noran System.

Os ensaios mecânicos de tração foram realizados em uma máquina universal Instron 5567 equipada com uma célula de carga de $1 \mathrm{kN}$. Os ensaios foram baseados na norma de teste padrão da ASTM (D 882-95a) para ensaios de tração em filmes poliméricos finos. Para cada amostra foram utilizados 5 corpos de prova, que foram previamente mantidos sob atmosfera controlada ( $43 \%$ de umidade relativa) por um período mínimo de uma semana. As condições climáticas da sala em que foram realizados os ensaios foram mantidas a uma umidade relativa de $(43 \pm 2) \%$ e temperatura de $(25 \pm 2){ }^{\circ} \mathrm{C}$. Os corpos de prova tinham o formato de fitas com 1,0 cm de largura, $4,0 \mathrm{~cm}$ de comprimento e espessura média de $(0,250 \pm 0,046) \mathrm{mm}$. A distância entre garras do equipamento foi configurada em $1,0 \mathrm{~cm}$ para que os filmes permanecessem afixados às garras pneumáticas durante o ensaio. Utilizou-se uma velocidade de ensaio de $5 \mathrm{~mm}$ $\mathrm{min}^{-1}$. Os resultados foram analisados através de curva de tensão versus deformação, de onde foram extraídos a tensão de ruptura $\left(\sigma_{U}\right)$ e o módulo de elasticidade $(\mathrm{E})$, este último através do ajuste da curva na seção linear inicial até $1 \%$ de deformação, pelo método dos mínimos quadrados.

\section{Resultados e Discussões}

\subsection{Imagens macroscópicas dos filmes}

A Figure 2 apresenta imagens obtidas com uma câmera digital, dos sólidos precursores e dos materiais híbridos obtidos.

Observa-se que os materiais híbridos após a reação mecanoquímica da paligorsquita com o sal do corante alaranjado de metila, na presença de $\mathrm{HCl}$ (PGS/AM) gerou uma coloração vermelho bordô, característica do corante em sua forma protonada e durante o tratamento térmico a coloração se tornou gradualmente marrom terra. Após o

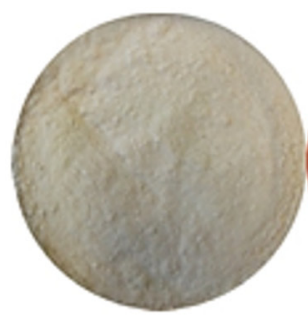

(a)

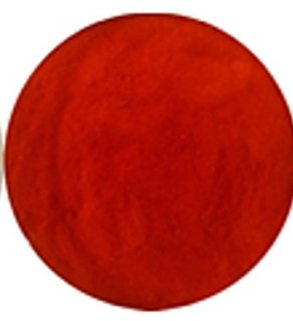

(b)

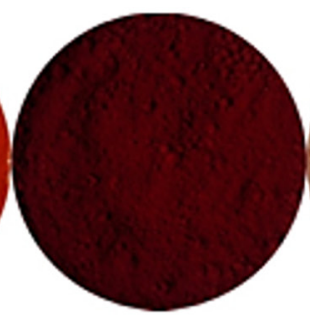

(c)

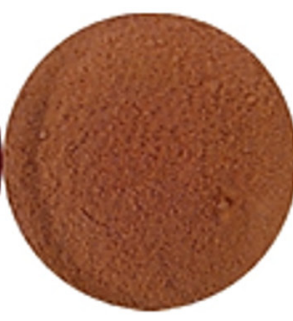

(d)

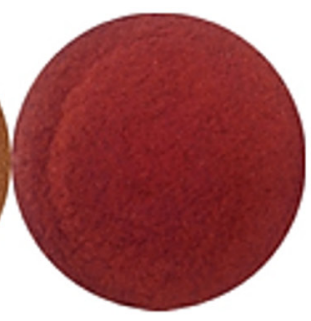

(e)

Figura 2. Imagem dos materiais de partida e dos materiais híbridos: Paligorsquita (a), Alaranjado de metila (b), Vermelho congo (c), PGS/AM (d), PGS/VC (e). 
processo de extração com a mistura de solvente etanol/água 9:1 (v/v), o material híbrido apresentou uma coloração laranjada característica da forma do corante desprotonado (Figure 2d). As intensidades das diferentes tonalidades da cor após o tratamento térmico e extração por Soxhlet podem ser atribuídas ao excesso dos corantes nos materiais, ou pelas diferentes formam com que interagem com a paligorsquita.

Pela mesma razão o material híbrido com o sal do corante vermelho congo na presença de $\mathrm{HCl}$ (Figure 2e) apresentou variação na coloração, mantendo a tonalidade azul no processo de reação mecanoquímica e no tratamento térmico. No processo de extração retornou a coloração característica do sal de sódio do corante vermelho congo com uma menor intensidade. Observa-se que ambos os materiais híbridos mantiveram a coloração após o processo de aquecimento e extração, demonstrando a efetiva interação desses com a estrutura da paligorsquita.

\subsection{Espectroscopia eletrônica na região do ultravioleta/ visível.}

Na Figure 3 são observados conjunto de espectros eletrônicos no modo de reflectância difusa dos materiais híbridos e seus respectivos precursores: material híbrido com alaranjado de metila (A) e espectros com o material híbrido vermelho congo (B).

A faixa espectral investigada do sal do corante Na-AM (Figure 3A-a) apresentou três bandas, duas na região ultravioleta $(258,328 \mathrm{~nm})$ e uma na região do visível $428 \mathrm{~nm}$, as mesmas não são observadas no material híbrido formado PGS/AM (Figure 3A-b), com exceção da banda em $258 \mathrm{~nm}$ que está relacionada com a ligação Si-O na estrutura da paligorsquita.

O material híbrido PGS/AM (Figure 3A-b), ainda apresenta uma banda em $490 \mathrm{~nm}$ que pode estar associada a possíveis interações do corante alaranjado de metila com a paligorsquita. De forma análoga o sal do corante vermelho congo (Figure 3B-a) apresentou três bandas de maior intensidade, sendo uma na região do ultravioleta $(258 \mathrm{~nm})$ e duas na região do visível (426 nm e $549 \mathrm{~nm}$ ).

Observa-se também uma banda na região do visível em $502 \mathrm{~nm}$ no espectro do material híbrido PGS/VC (Figure 3B-b), sugerindo que o corante imobilizado no material híbrido está na forma desprotonada, o qual em solução apresenta uma banda na região de 498 nm, caracterizando a coloração vermelha.

Como mencionado anteriormente, a paligorsquita não apresenta nenhuma banda de absorção na região do visível, sendo atribuídas então as bandas na região de $333 \mathrm{~nm}$ e $504 \mathrm{~nm}$ do material híbrido PGS/VC para anéis naftalênicos e as ligações azo presentes na estrutura do corante. As bandas do corante em solução aquosa são observadas em $333 \mathrm{~nm}$ e 498 nm.Para determinar o teor de imobilização (loading) das moléculas orgânicas na paligorsquita, os teores de material extraído pelo método de Soxhlet foram analisador por UV-Vis, monitorando-se o sal de AM em $420 \mathrm{~nm}$ e no sal do VC em $520 \mathrm{~nm}$.

Percentuais de imobilização de 99\% e 100\% foram obtidos nos compostos híbridos PGS/AM e PGS/VC, respectivamente $(4 \%$ em massa em relação à massa da paligorsquita seca).

Observou-se que um fator determinante na imobilização dos corantes no argilomineral é a realização da síntese mecanoquímica em meio de solução ácida, obtendo valores maiores de imobilização, uma vez que a síntese dos materiais híbridos em solução aquosa neutra, onde um maior percentual de massa do corante foi utilizado (8\%), apresentou uma percentual de imobilização inferior a $2 \%$.

A fim de avaliar a estabilidade química dos materiais híbridos recém-sintetizados utilizou um método de Van Ohphen, com pequenas adaptações ${ }^{[8]}$. Esse método tem com objetivo a distinção de originalidade do pigmento Azul Maya a partir de outros pigmentos semelhantes. No estudo de estabilidade química dos materiais híbridos obtidos utilizaram-se soluções aquosas neutras, alcalinas (hidróxido de sódio $0,5 \mathrm{~mol} / \mathrm{L}$ ) e ácidas (ácido nítrico $0,5 \mathrm{~mol} / \mathrm{L}$ ). No procedimento adotado, os materiais híbridos foram adicionados nas diferentes soluções por um período de 24 horas, sob agitação magnética. Após este período as dispersões foram centrifugados a $9000 \mathrm{rpm}$ com uma força $\mathrm{g}$ de 7606 por um período de 30 minutos e sobrenadante foi monitorado pela técnica UV-Vis, empregando curvas padrões dos corantes em diferente regiões conforme a solução utilizada.
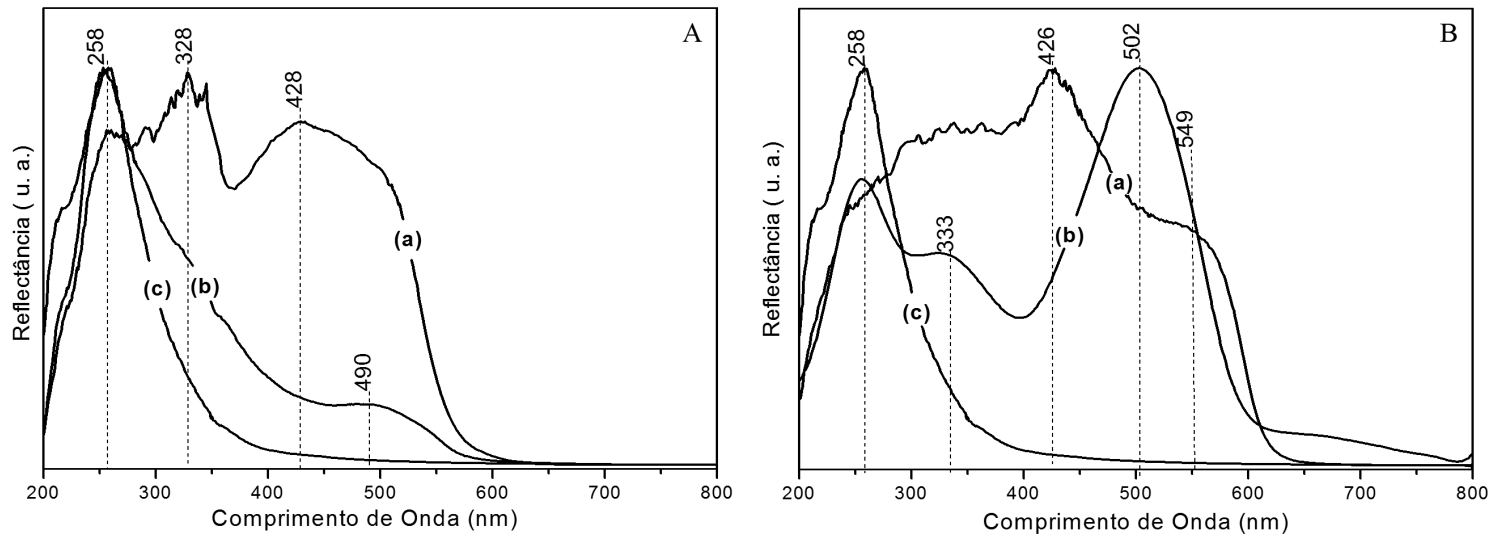

Figura 3. Espectros de reflectâcia difusa de UV-VIS (normalizados) dos precursores: Alaranjado de metila (A-a), vermelho congo (B-a), Paligorsquita (A-c, B-c) e dos materiais híbridos, PGS/AM (A-b), PGS/VC (B-b). 
O corante alaranjado de metila em meio de solução alcalina foi monitorado na região de $420 \mathrm{~nm}$ e $505 \mathrm{~nm}$ em meio de solução ácida. Já o corante vermelho congo foi monitorado na região de $399 \mathrm{~nm}$, em meio de solução alcalina e na região de $660 \mathrm{~nm}$ em meio de solução ácida. Em solução aquosa neutra, os corantes foram monitorado na região de $462 \mathrm{~nm}$ e $498 \mathrm{~nm}$ para o alaranjado de metila e vermelho congo, respectivamente. Observou-se que somente em meio alcalino houve liberação de cerca $0,05 \%$ do corante alaranjado de metila para o material híbrido PGS/AM e 0,10\% de corante vermelho congo para o material híbrido PGS/VC.

Em meio neutro e ácido, os teores de corante liberado foram abaixo dos limites de detecção da técnica. Esses experimentos comprovam que as interações entre os corantes e a matriz de paligorsquita são fortes, não sendo destruídas nem em condições agressivas de pH ácido ou alcalino. Essa característica é bastante desejável e certamente os sistemas estudados simulam condições próximas do pigmento Azul Maya, que apresenta estabilidade química semelhante.

\subsection{Caracterizações texturais}

No que consiste às caracterizações texturais, as isotermas de adsorção/dessorção de nitrogênio (Material Suplementar, Figura 1S) demonstram que todos os materiais sejam eles na forma "in natura", tratada ou híbrida, apresentam o mesmo comportamento, sendo as isotermas classificadas como do tipo II com histerese do tipo H3, correspondente a um material mesoporoso segundo a classificação da IUPAC ${ }^{[19,20]}$. Observa-se também que em todos os materiais ocorreu uma baixa adsorção em pressões relativas inferiores a 0,45 , o que caracteriza uma superfície pobre em microporos. Entretanto quando a pressão relativa é superior a 0,45 começa ocorrer um aumento da adsorção, separando as curvas, comprovando que todos os materiais são sólidos mesoporosos.

Na Tabela 1 são apresentas as áreas superficiais referentes os argilominerais "in-natura", e dos materiais híbridos.

Observa-se que todos os materiais apresentam um pequeno aumento na área superficial especifica na área externa e no volume de poro quando comparado com o argilomineral "in-natura”. Este aumento está relacionado a imobilização de compostos orgânicos na estrutura dos argilominerais podendo levar a uma formação de redes bi-dimensionais de ordem de micro ou mesoporo ${ }^{[21]}$. O material híbrido contendo o corante vermelho congo imobilizado apresentou um aumento de área de $27,9 \mathrm{~m}^{2} \mathrm{~g}^{-1}$ em relação ao argilomineral "in-natura" o que pode sugerir que o corante pode estar interagindo com diferentes fibrilas, através dos dois grupos sulfonatos nas partes extremas da molécula e ao separá-las produz um volume interfibrilar extra.

Essas variações da área externa e área superfícial podem sugerir diferentes modos de imobilização do corante na superfície e nos poros da estrutura do argilomineral. Baseado nas dimensões dos poros da paligorsquita e nas dimensões dos corantes, somente o ânion derivado do alaranjado de metila teria condições de ser totalmente inserido nos poros da paligorsquita. Já o material híbrido com o corante vermelho congo somente parte do ânion poderia ser inserida nos poros do argilomineral (Figure 4).

Tabela 1. Propriedades texturais dos materiais avaliados.

\begin{tabular}{|c|c|c|c|}
\hline Amostra & $\begin{array}{c}\text { Área superficial } \\
\operatorname{BET}\left(\mathrm{m}^{2} \cdot \mathrm{g}^{-1}\right)\end{array}$ & $\begin{array}{c}\text { Área Externa } \\
\text { t-method }\left(\mathrm{m}^{2} \cdot \mathrm{g}^{-1}\right)\end{array}$ & $\begin{array}{c}\text { Volume de poro } \\
\left(\mathrm{cm}^{3} \cdot \mathrm{g}^{-1}\right)\end{array}$ \\
\hline PGS & 125,3 & 102,08 & 34,33 \\
\hline PGS/AM & 130,5 & 110,50 & 46,32 \\
\hline PGS/AVC & 153,2 & 122,08 & 62,48 \\
\hline
\end{tabular}
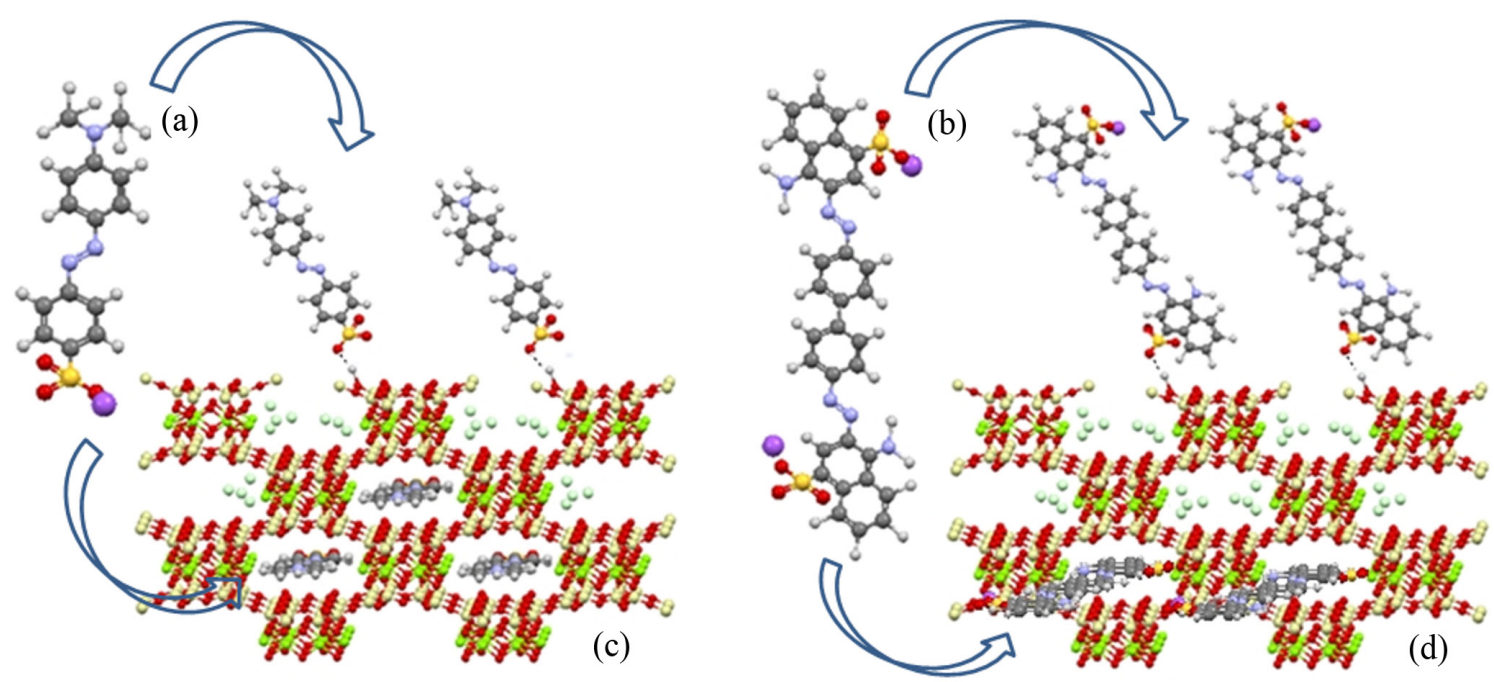

Figura 4. Imagem ilustrativa do corante azo: alaranjado de metila (a) e vermelho congo (b) e dos materiais híbridos, PGS/AM (c), PGS/VC (d). 


\subsection{Imagens dos filmes obtidos.}

A paligorsquita foi utilizada como carga no PVA para estudo comparativo, obtendo-se filmes homogêneos e transparentes mesmo com teores de $8 \%$ do argilomineral no PVA o que atesta a boa dispersão das cargas no polímero e a formação de nanocompósitos. Observa-se também uma leve tonalidade amarela em todos os filmes com o argilomineral "in-natura" (Figure 5A), a qual pode ser atribuída à presença de ferro presente como contaminante no argilomineral, como identificando pela técnica de EDS (Material Suplementar, Figura 2S).

Da mesma forma, os materiais híbridos contendo o corante alaranjado de metila (Figure 5B) e vermelho congo (Figure 5C) são coloridos e transparentes o que atesta a boa homogeneidade e manutenção do corante no argilomineral após a preparação dos filmes dos nanocompósitos. Observa-se que a intensificação da coloração ocorre com o aumento do teor de pigmento, conforme esperado para esse tipo de material.

\subsection{Caracterizações estruturais.}

Nos difratogramas de raios X dos filmes de PVA e dos nanocompósitos de PVA contendo os materiais híbridos ou argilomineral puro (Figure 6), com diferentes percentuais de carga, observa-se que todos os filmes apresentado um

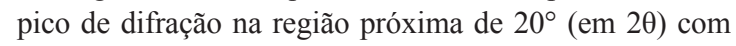

um pequeno alargamentos para os filmes com os materiais híbridos, e um ombro na região de $22,5^{\circ}$ (em $2 \theta$ ) que são correspondente aos planos 10-1 e 101 característico da estrutura cristalina do PVA atático ${ }^{[22,23]}$.

Nos filmes contendo o argilomineral na forma "in-natura" (Figure 6A) ou do material híbridos (Figure 6B, C), o pico de difração mais intenso da paligorsquita em $8,5^{\circ}$ (em 20) referente o plano 110 do argilomineral se manteve inalterado. Esse comportamento é o esperado já que se trata de um argilomineral fibroso com canais tridimensionais rígidos, não alterando seus parâmetros de rede após a inserção do corante ou de qualquer outra espécie. Os filmes ainda apresentam quartzo como contaminante, oriundo do argilomineral.

Nas imagens de microscopia eletrônica de varredura dos filmes (Material Suplementar, Figura 3S), observou que em todas as amostras com exceção do polímero puro, apresentaram fibrilas características da morfologia do argilomineral as quais estão dispersas no polímero, aparentemente de forma homogênea ao longo da sessão transversal do filme. Nas imagens observou também pontos escuros que são gerados durante o processo de medida e como são relativos a danos provocados pelo feixe de elétrons, devem ser desconsiderados. Nas medidas de EDS (Tabela 2) observou-se que todas as amostras apresentaram elementos característicos do argilomineral, além de um alto percentual de carbono e oxigênio referente à matriz polimérica.

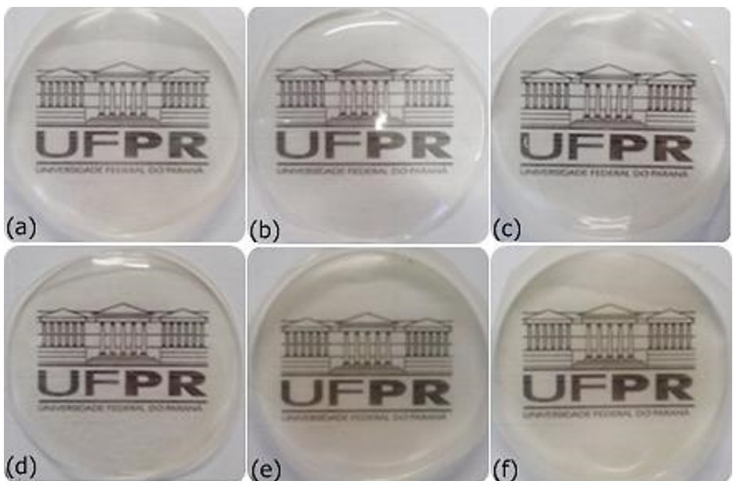

(A)

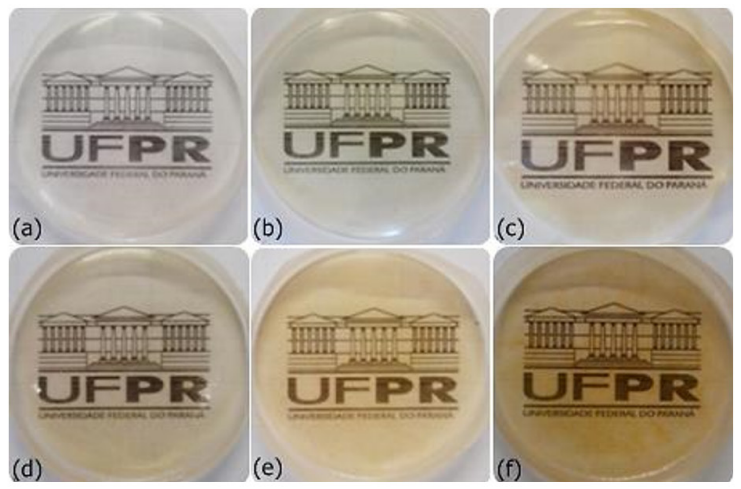

(B)

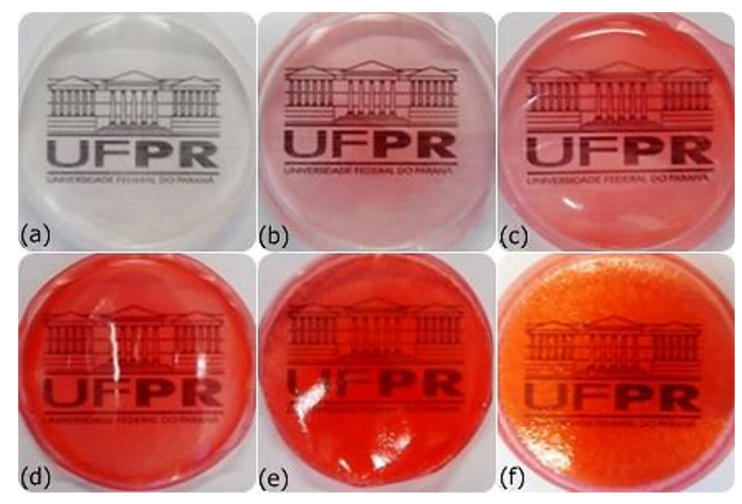

(C)

Figura 5. Fotografias digitais dos filmes de PVA contendo argilomineral (A) e materiais híbridos, PGS/AM (B) e PGS/VC (C), nas seguintes proporções: (a) $0 \%$ (PVA); (b) 1,0\%; (c) 2,0\%; (d) 3,0\%; (e) $5,0 \%$ e (f) $8,0 \%$. 


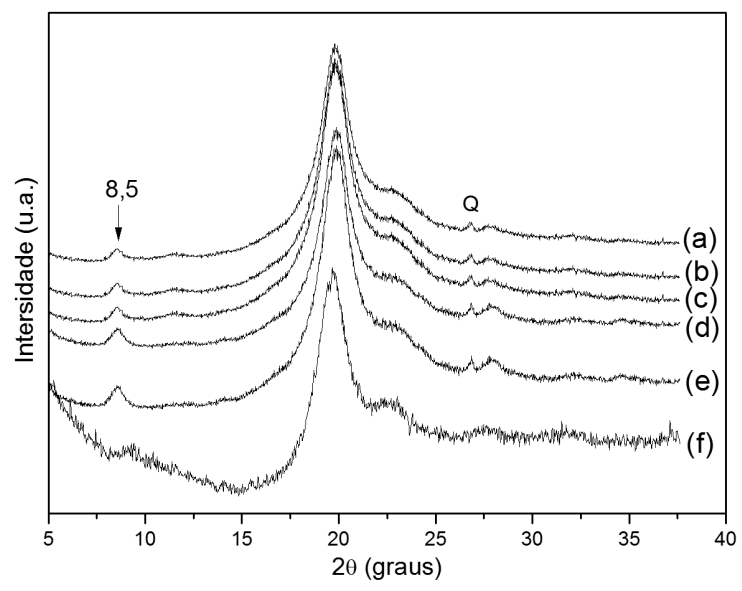

(A)

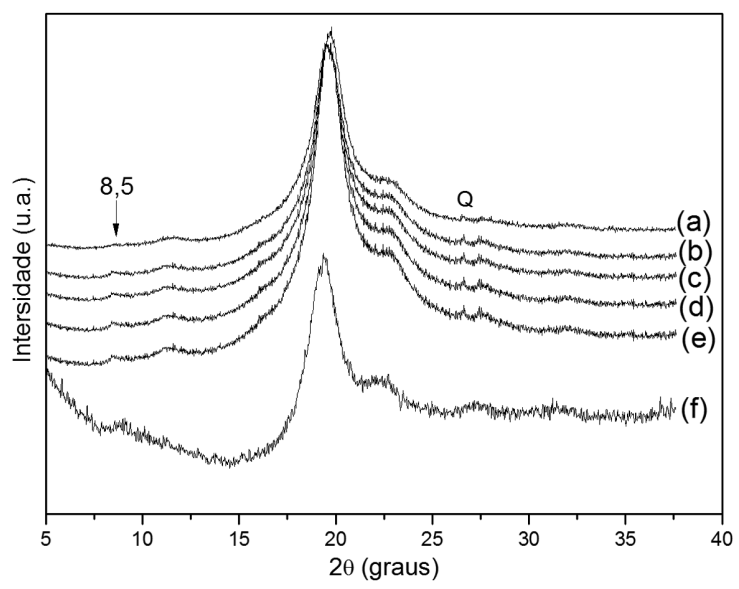

(B)

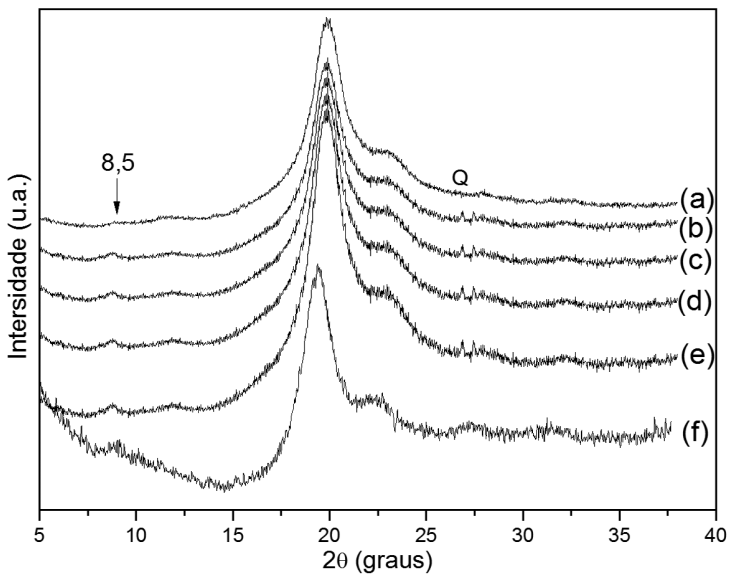

(C)

Figura 6. Difratogramas de raios $x$ dos filmes de PVA contendo o argilomineral (A), e dos materiais híbridos PGS/AM (B) e PGS/VC (C) com diferente percentagem em relação a massa do PVA: (a) 0\%; (b) 2\%; (c) 3\%; (d) 5\%; (e) 8\%; (f) 0\%. Todas as análises foram realizadas na parte superior dos filmes. Contaminante: $(\mathrm{Q}=$ quartzo).

Tabela 2. Resultados das análises de EDS dos filmes (\% em massa).

\begin{tabular}{cccccccc}
\hline Amostra & C (\%) & O (\%) & Mg (\%) & Al (\%) & Si (\%) & Ca (\%) & Na (\%) \\
\hline PVA & 77,73 & 21,59 & - & - & - & - & 0,68 \\
PVA-PGS 1\% & 72,21 & 21,37 & 0,29 & 1,69 & 2,06 & - & 2,38 \\
PVA-PGS 8\% & 72,31 & 19,93 & 0,42 & 2,84 & 0,96 & - & 3,54 \\
PVA-PGS/AM 1\% & 68,41 & 22,19 & 3,97 & 2,54 & 2,43 & 0,46 & - \\
PVA-PGS/AM 8\% & 73,83 & 20,98 & 1,36 & 1,60 & 2,24 & - & - \\
PVA-PGS/VC 1\% & 71,26 & 21,86 & 0,36 & 0,36 & 0,67 & 1,05 & 4,44 \\
PVA-PGS/VC 8\% & 65,40 & 22,78 & 0,75 & 1,08 & 3,36 & - & 6,63 \\
\hline
\end{tabular}

\subsection{Propriedades mecânicas}

Os resultados das propriedades mecânicas se referem às medidas de módulo de Young, tensão máxima e alongamento dos filmes de PVA puro e nanocompósitos contendo a paligorsquita pura e os materiais híbridos (Figure 7). Os valores obtidos pelos ensaios mecânicos com suas respectivas médias e desvio padrão são referentes às médias de cinco amostras testadas para cada filme. Embora os nanocompósitos se apresentarem com boa homogeneidade ao longo da sessão transversal, existe um grande desvio padrão na maioria das medidas de módulo o que pode ser sido ocasionado pela pequena flutuação na espessura dos filmes, os quais foram obtidos por evaporação em placa de Petri. Observa-se que especialmente nas laterais da placa de Petri, os filmes são levemente mais espessos.

Observa-se que a paligorsquita “in-natura” e a material híbrido PVA-PGS/VC mostra pouca influência nas propriedades elásticas do PVA. O mesmo efeito é observado para a tensão máxima, com tendência para a diminuição dessa propriedade. Apesar da manutenção do módulo, as amostras tendem a diminuir o alongamento para o híbrido PVA-PGS/VC embora essa propriedade esteja próxima do 


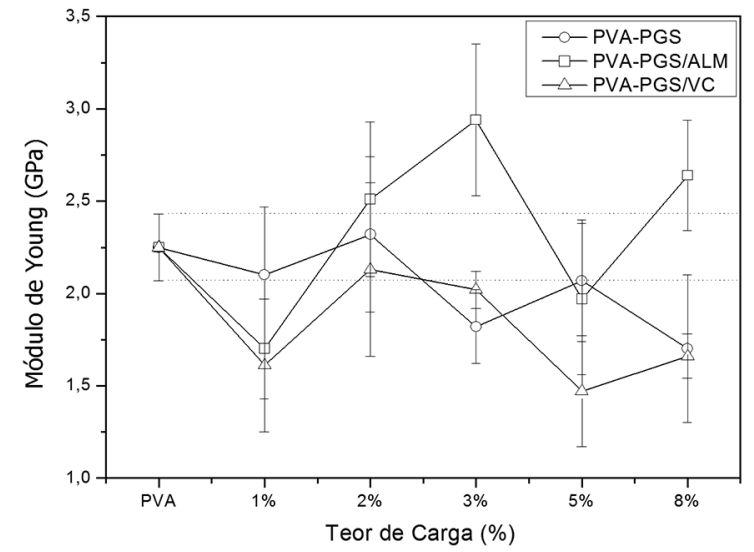

(a)

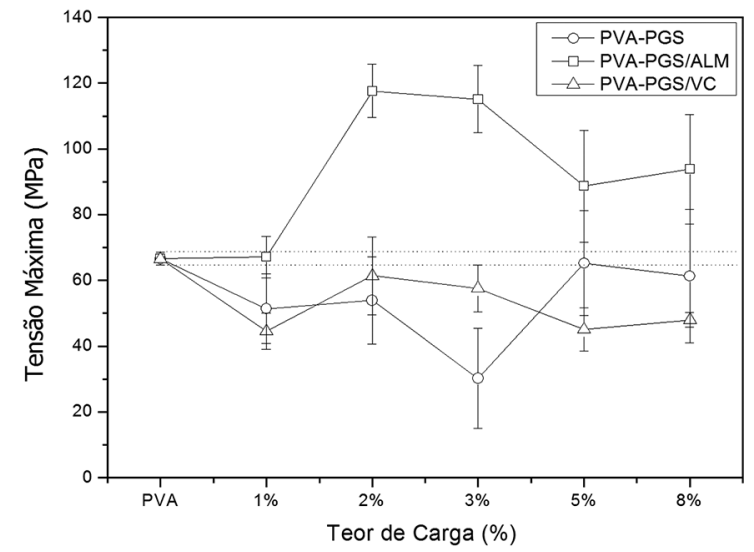

(b)

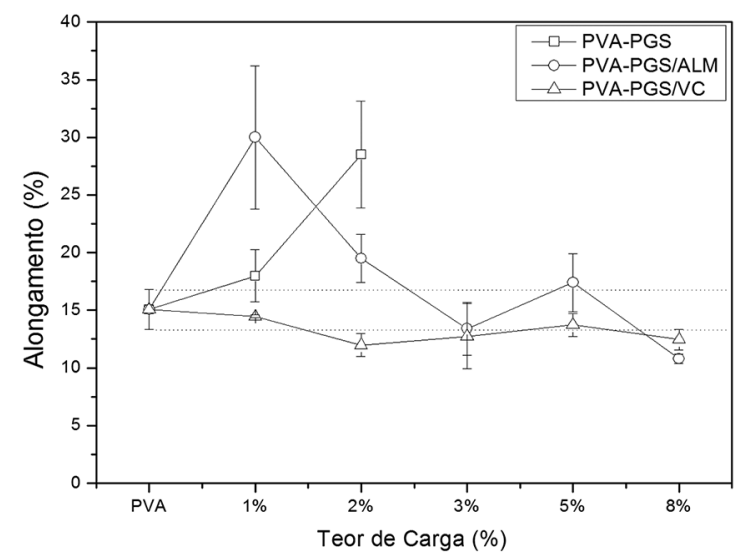

(c)

Figura 7. Módulo de Young (a), tensão máxima (b) e alongamento (c) dos filmes de PVA contendo diferentes teores materiais híbridos ou argilomineral. * - O alongamento das amostras de PVA-PGS após $2 \%$ de carga não foram registrados por problemas no equipamento.

valor da flutuação do erro da medida o que limita qualquer conclusão a esse respeito.

A paligorsquita é hidrofílica e deveria permitir uma boa interação com a matriz hidrofílica do PVA, e uma boa adesão interfacial entre a superfície das fibras do argilomineral com as moléculas de polihidroxiladas do PVA, restringindo o movimento molecular durante o alongamento das amostras porém as fibrilas tendem a atuar mais como um plastificante do que como um reforço.

No caso da utilização da carga PVA-PGS/AM, com pequenos teores de 2 e $3 \%$, existe um aumento significativo do aumento do módulo, o que atesta uma boa interação da carga com o polímero. Em todos os teores de carga, ocorreu um aumento da tensão máxima, o que é muito desejável já que as amostras embora mais rígidas, são mais resistentes à tensão aplicada. O pico da tensão máxima ocorre para os teores de 2 e $3 \%$ de carga, fato comum para os nanocompósitos poliméricos reforçados com argilominerais. $\mathrm{O}$ alongamento normalmente possui um comportamento que é o inverso do módulo, quando maior o módulo, as amostras são mais rígidas e possuem uma menor capacidade de alongamento, conforme observado.

\section{Conclusões}

Os materiais híbridos (paligorsquita contendo os corantes azo alaranjado de metila e vermelho congo) foram obtidos através de reação mecanoquímica em meio ácido, obtendo-se um teor próximo de 4\% de imobilização dos corantes no argilomineral.

Foi possível identificar a presença dos corantes azo nos diferentes materiais híbridos formados utilizando a técnica de espectroscopia eletrônica na região do ultravioleta-visível, sugerindo-se que o corante encontra-se em sua forma desprotonada após a extração do excesso dos corantes.

Estudos de lixiviação demonstraram que somente em meio fortemente alcalino uma pequena liberação dos corantes azo utilizados foi constatada, sugerindo que os materiais híbridos são estáveis à ataques químicos à exemplo do pigmento Azul Maya.

As medidas texturais demostraram que os materiais híbridos, além do argilomineral "in-natura", são materiais mesoporosos, sendo que os materiais híbridos apresentam uma maior área especifica, característica de formação de pontes de corantes que conectam diferentes fibras (fibrilação).

Os filmes dos nanocompósitos de PVA apresentaram uma boa dispersão das cargas na matriz poliméricas, sendo 
evidente na homogeneidade, na coloração e transparência. Os filmes foram caracterizados por difração de raios X (DRX) e apresentaram picos de difração característicos da presença do argilomineral além de picos da matriz polimérica. Com as micrografias e dos dados das medidas e de espectroscopia de energia dispersiva, foi possível comprovar a presença das cargas com uma boa homogeneidade ao longo da sessão transversal das fraturas desses filmes.

Nos ensaios mecânicos de tração pode-se observar que os materiais nanocompósitos contendo o material híbrido PVA-PGS/AM tendem a aumentar o módulo e a tensão máxima e diminuir o alongamento o que atesta uma boa interação das fibras contendo o alaranjado de metila, em relação à paligorsquita "in natura" e o material híbrido PVA-PGS/VC. Além desse aumento das propriedades mecânicas, especialmente para os teores de 2 e $3 \%$ ocorre a atribuição de novas funções no nanocompósito de PVA como a cor, transparência, absorção da radiação ultravioleta e potencialmente outras não investigadas.

\section{Agradecimentos}

Os autores gostariam de agradecer as agências de fomento CNPq, FINEP, CAPES e Projeto Pronex Nennan (Fundação Araucária/CNPq). Agradecimentos também ao Centro de microscopia eletrônica da UFPR, pelas análises de $\mathrm{MEV} / \mathrm{EDS}$.

\section{Referências}

1. Usuki, A., Kawasumi, M., Kojima, Y., Okada, A., Kurauchi, T., \& Kamigaito, O. (1993). Swelling behavior of montmorillonite cation exchanged for $\omega$-amino acids by $\mathrm{C}$-caprolactam. Journal of Materials Research, 8(5), 1174-1178. http://dx.doi. org/10.1557/JMR.1993.1174.

2. Kojima, Y., Usuki, A., Kawasumi, M., Okada, A., Fukushima, Y., Kurauchi, T., \& Kamigaito, O. (1993). Mechanical properties of nylon 6-clay hybrid. Journal of Materials Research, 8(5), 1185-1189. http://dx.doi.org/10.1557/JMR.1993.1185.

3. Lombardo, P. C., Poli, A. L., \& Schitt, C. C. (2015). Influência de estabilizantes na degradação foto-oxidativa de filmes de compósitos de SWy-1/poli(óxido de etileno). Polímeros: Ciência e Tecnologia, 25(1), 101-108. http://dx.doi.org/10.1590/01041428.1604.

4. Lecouvet, B., Sclavons, M., Bourbigot, S., \& Bailly, C. (2013). Thermal and flammability properties of polyethersulfone/ halloysite nanocomposites prepared by melt compounding. Polymer Degradation \& Stability, 98(10), 1993-2004. http:// dx.doi.org/10.1016/j.polymdegradstab.2013.07.013.

5. Cavalcanti, W. S., Brito, G. F., Agraval, P., Melo, T. J. A., Neves, G. A., \& Dantas, M. M. (2014). Purification and organophilization in pilot scale of bentonitic clays with a nonionic surfactant and their application in polymer nanocomposites. Polímeros: Ciência e Tecnologia, 24(4), 491-500. http://dx.doi. org/10.1590/0104-1428.1539.

6. Komatsu, D., Otaguro, H., \& Ruvolo Filho, A. (2014). Avaliação comparativa entre os nanocompósitos de argila motmorilonita/LLDPE e com hexaniobato de potássio/LLDPE: caracterização das propriedades mecânicas e de transporte. Polímeros: Ciência e Tecnologia, 24(1), 37-44. http://dx.doi org/10.4322/polimeros.2013.052.

7. Arnold, D. E. (2005). Maya blue and Palygorskite: a second possible pre-Columbian source. Ancient Mesoamerica, 16(1), 51-62. http://dx.doi.org/10.1017/S0956536105050078.
8. Van Olphen, H. (1966). Maya blue: a clay organic pigment? Science, 154(3749), 645-646. http://dx.doi.org/10.1126/ science.154.3749.645. PMid:17778806.

9. Kleber, R., Masschelein-Kleiner, L., \& Thissen, J. (1967). Study and identification of Maya blue. Studies in Conservation, 12(1), 41-56.

10. Giustetto, R., Seenivasan, K., Pellerej, D., Ricchiardi, G., \& Bordiga, S. (2012). Spectroscopic characterization and photo/ thermal resistance of a hybrid palygorskite/methyl red Mayan pigment. Microporous and Mesoporous Materials, 155(1), 167-176. http://dx.doi.org/10.1016/j.micromeso.2012.01.024.

11. Giustetto, R., \& Wahyudi, O. (2011). Sorption of red dyes on palygorskite: Synthesis and stability of red/purple Mayan nanocomposites. Microporous and Mesoporous Materials, 142(1), 221-235. http://dx.doi.org/10.1016/j.micromeso.2010.12.004.

12. Chen, H., \& Zhao, J. (2009). Adsorption study for removal of Congo red anionic dye using organo-attapulgite. Adsorption, 15(4), 381-389. http://dx.doi.org/10.1007/s10450-009-9155-z.

13. Zhang, Y., Wang, W., Zhang, J., Liu, P., \& Wang, A. (2015). A comparative study about adsorption of natural palygorskite for methylene blue. Chemical Engineering Journal, 262(1), 390-398. http://dx.doi.org/10.1016/j.cej.2014.10.009.

14. Del Rio, M. S., Martinetto, P., Reyes-Valerio, C., Dooryhee, E., \& Suarez, M. (2006). Synthesis and acid resistance of Maya Blue pigment. Archaeometry, 48(1), 115-130. http://dx.doi. org/10.1111/j.1475-4754.2006.00246.x.

15. Marangoni, R., Gardolinski, J. E. F C., Mikowski, A., \& Wypych, F. (2011). PVA nanocomposites reinforced with $\mathrm{Zn}_{2} \mathrm{Al}$ LDHs, intercalated with orange dyes. Journal of Solid State Electrochemistry, 15(2), 303-311. http://dx.doi.org/10.1007/ s10008-010-1056-2.

16. Marangoni, R., Ramos, L. P., \& Wypych, F. (2009). New multifunctional materials obtained by the intercalation of anionic dyes into layered zinc hydroxide nitrate followed by dispersion into poly(vinyl alcohol) (PVA). Journal of Colloid and Interface Science, 330(2), 303-309. http://dx.doi. org/10.1016/j.jcis.2008.10.081. PMid:19081109.

17. Zimmermann, A., Jareger, S., Zawadzki, S. F., \& Wypych, F. (2014). Nanocompósitos poliméricos de polietileno de alta densidade contendo hidróxidos duplos lamelares intercalados com anions derivados de corantes azo. Polímeros: Ciência e Tecnologia, 24(3), 332-343. http://dx.doi.org/10.4322/ polimeros.2014.025.

18. da Silva, M. L. N., Marangoni, R., da Silva, A. H., Schreiner, W. H., \& Wypych, F. (2013). Poly(vinyl alcohol) composites containing layered hydroxide salts, intercalated with anionic azo dyes (Tropaeolin 0 and Tropaeolin 0), Polímeros: Ciência e Tecnologia, 23(2), 248-256. http://dx.doi.org/10.1590/S010414282013005000026 .

19. Sing, K. S. W., Everett, D. H., Haul, R., Moscou, L., Pierotti, R. A., Rouguerol, J., \& Siemieniewska, T. (1985). Reporting physisorption data for gas/solid systems with special reference to the determination of surface area and porosity (recommendations 1984). Pure and Applied Chemistry, 57(4), 603-619. http:// dx.doi.org/10.1351/pac198557040603.

20. Boudriche, L., Calvet, R., Hamdi, B., \& Balard, H. (2011). Effect of acid treatment on surface properties evolution of attapulgite clay: an application of inverse gas chromatography. Colloids and Surfaces. A, Physicochemical and Engineering Aspects, 392(1), 45-54. http://dx.doi.org/10.1016/j.colsurfa.2011.09.031.

21. Wang, C. C., Juang, L. C., Lee, C. K., Hsu, T. C., Lee, J. F., \& Chao, H. P. (2004). Effects of exchanged surfactant cations on the pore structure and adsorption characteristicsof montmorillonite. Journal of Colloid and Interface Science, 280(1), 27-35. http://dx.doi.org/10.1016/j.jcis.2004.07.009. PMid: 15476770. 
22. Ricciardi, R., Auriemma, F., De Rosa, C., \& Laupretre, F. (2004). $\mathrm{X}$-ray diffraction analysis of poly(vinyl alcohol) hydrogels, obtained by freezing and thawing techniques. Macromolecules, 37(5), 1921-1927. http://dx.doi.org/10.1021/ma035663q.

23. Garcia-Cerda, L. A., Escareno-Castro, M. U., \& SalazarZertuche, M. (2007). Preparation and characterization of polyvinyl alcohol-cobalt ferrite nanocomposites. Journal of
Non-Crystalline Solids, 353(8-10), 808-810. http://dx.doi. org/10.1016/j.jnoncrysol.2006.12.046.

Enviado: Maio 15, 2015

Revisado: Jul. 29, 2015

Aceito: Ago. 31, 2015 


\section{Material Suplementar}

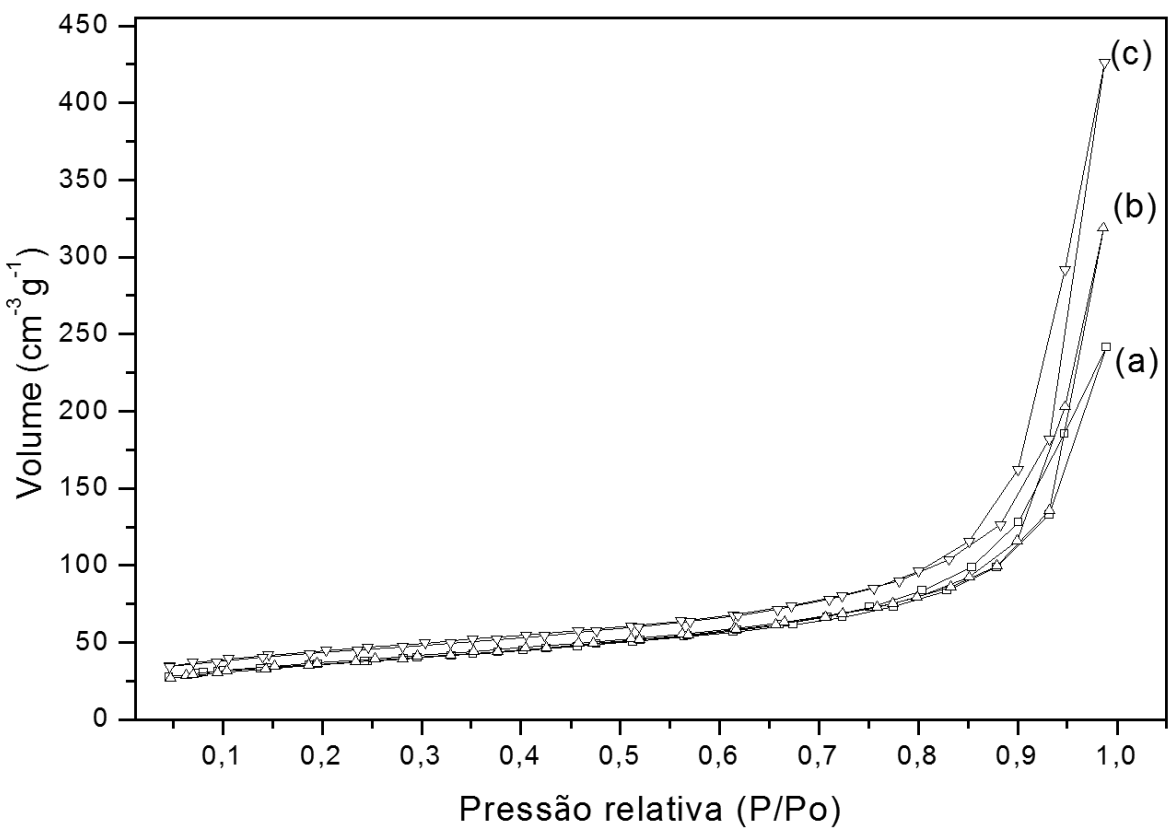

Figura 1S. Isoterma de adsorçâo/desorção de nitrogênio da paligorsquita (a) e dos materiais híbridos PGS/AM (b) e PGS/VC (c).
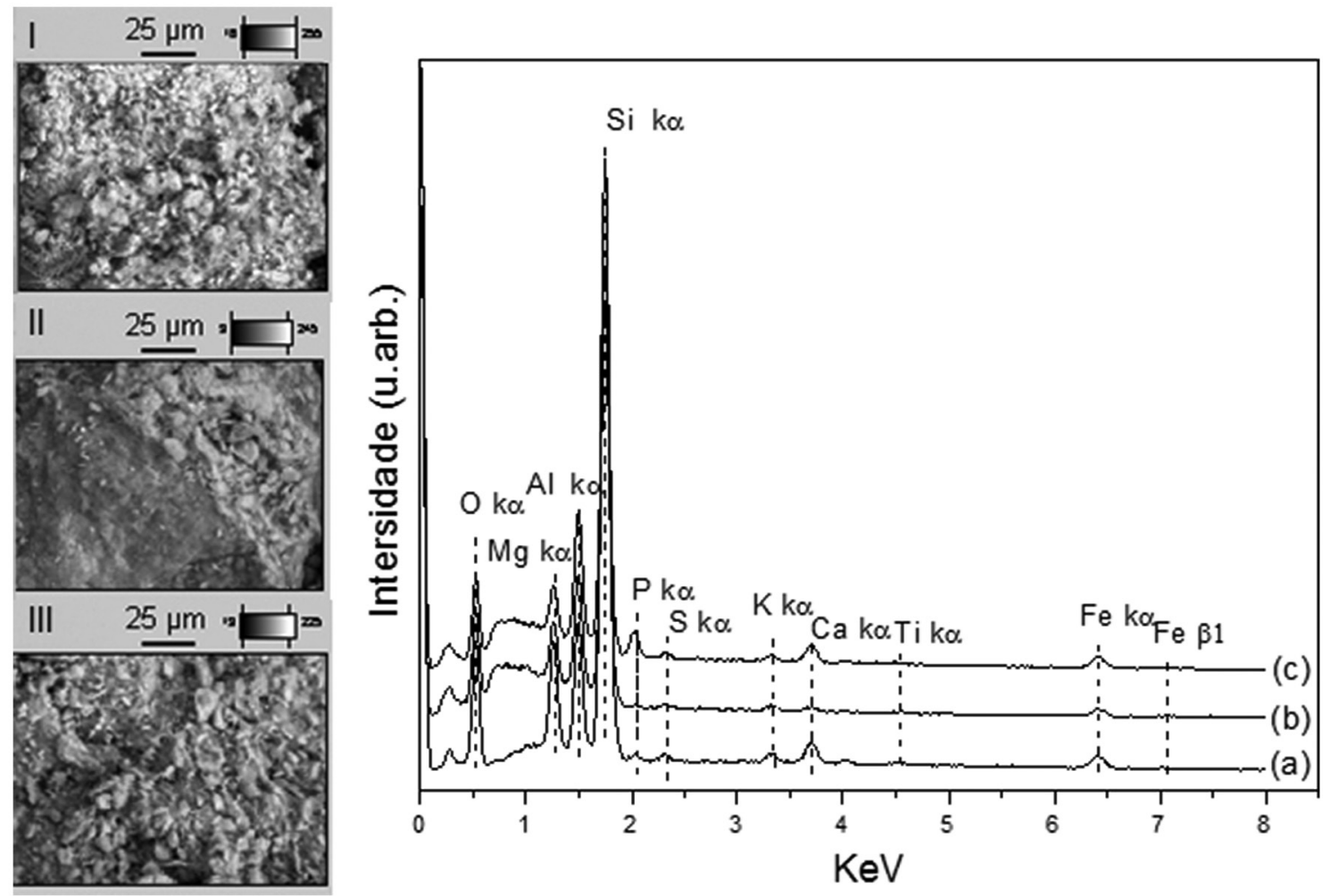

Figura 2S. Imagens de MEV e espectro de EDS do argilomineral "in-natura" (I-a), e dos materiais híbridos PGS/AM (II-b) e PGS/VC (III-c). Voltagem $15 \mathrm{kv}$ e ampliação de 5000x. 

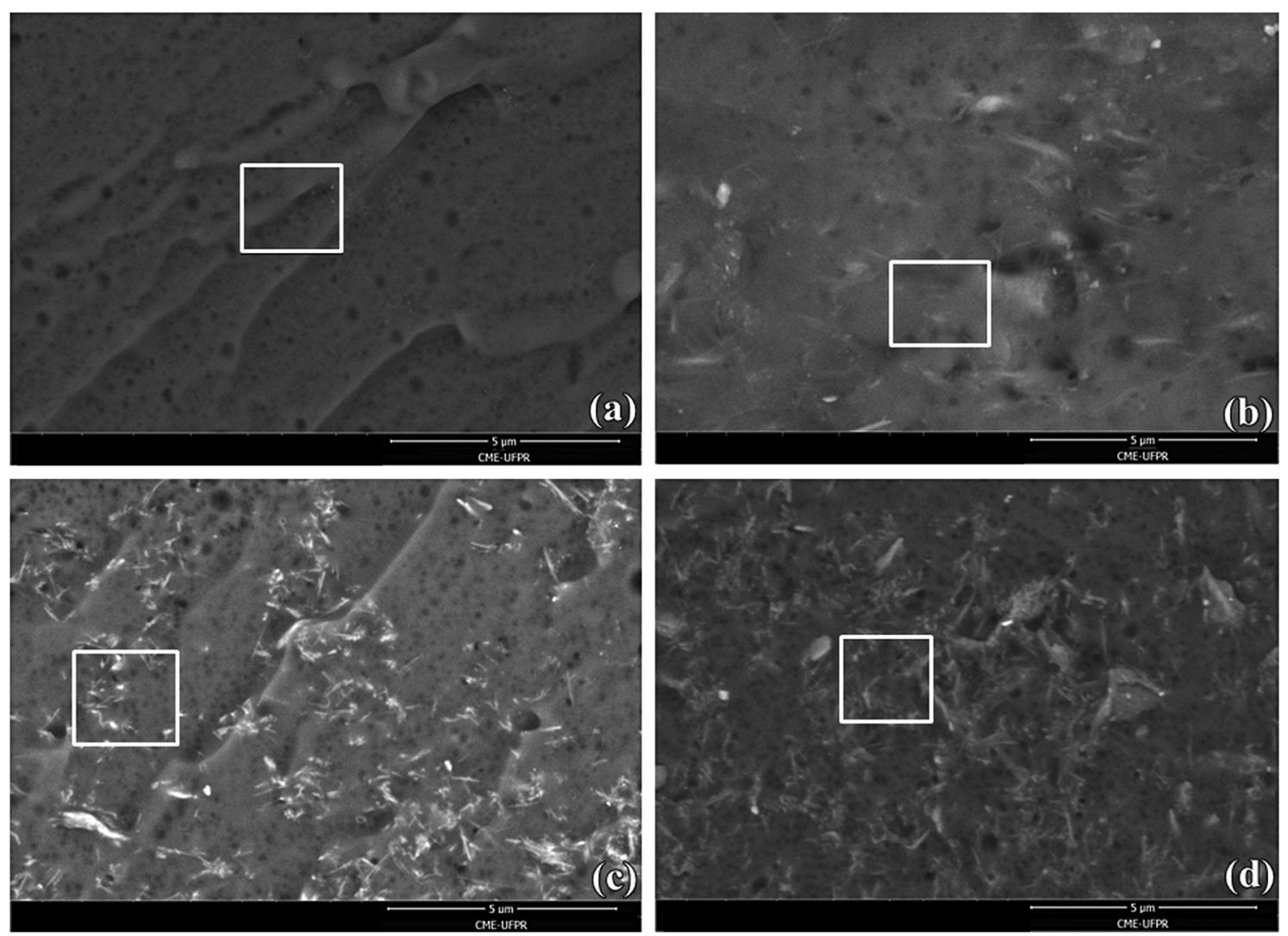

Figura 3S. Micrografias dos filmes na secção transversal: PVA puro (a) e dos filmes contendo (8\%) em carga: argilomineral "in-natura" (b), PGS/AM (c) e PGS/VC (d). 